\title{
Interactions à longue portée et systèmes non extensifs
}

Jacques Villain (Jacques.Villain@cea.fr), Directeur de recherche honoraire au CEA de Grenoble

Si des interactions à

longue portée

(telle que l'interaction

gravitationnelle) sont

présentes dans un milieu,

certaines lois classiques

de la thermodynamique

peuvent être violées.

Par exemple, l'énergie

n'est plus proportionnelle,

à densité constante,

au nombre de particules.

Plus grave, certains états

instables à l'équilibre

avec un thermostat

(situation " canonique ")

peuvent devenir stables

pour un système isolé

(situation " microcano-

nique »). À l'équilibre

microcanonique,

la chaleur spécifique

peut devenir négative.

Nous analysons

brièvement

ces paradoxes en termes

simples à l'usage du

non-spécialiste.

\section{Introduction}

"L'énergie $U$ et l'entropie $S$ sont additives ; ceci veut dire que lorsque le nombre de particules $N$ varie, $U$ et $S$ sont proportionnelles à $N$. " C'est essentiellement ce qu'on lit (\$24) dans le traité de physique statistique de Landau et Lifshitz [1].

Or l'additivité (ou " extensivité ») n'est en général pas vérifiée pour des interactions à longue portée (voir encadré p. 11) telles que les interactions gravitationnelles. Les progrès récents de l'astronomie ont provoqué un renouveau d'intérêt pour la mécanique statistique non extensive [2]. Mais on connaît depuis longtemps [3] la non-extensivité dans le noyau atomique, due aux forces de répulsion coulombiennes entre protons.

Si des interactions à longue portée sont présentes, il peut arriver que certains concepts fondamentaux soient en défaut. Ainsi, la capacité calorifique peut-elle (dans des conditions très particulières) devenir négative [4]. Le présent article s'efforce de trier l'essentiel de ce qui reste valable dans la thermodynamique de nos manuels, et ce qui doit être revu quand les interactions sont à longue portée. Nous choisirons la simplicité du raisonnement au détriment de la rigueur et nous utiliserons des modèles non physiques quand ils peuvent aider à la compréhension des mécanismes.

\section{Concepts fondamentaux de la mécanique statistique}

Ces concepts, exposés dans les manuels [5], seront rappelés brièvement ici dans le cas d'un système physique ayant un volume $V$ donné, un nombre de particules $N$ donné et (conformément aux règles de la mécanique quantique) des états discrets $i$ d'énergie $E_{i}$. La mécanique statistique repose sur une expression de la probabilité $p_{i}$ que ce système soit dans l'état $i$. Pour un tel système, il existe deux expressions classiques de $p_{i}$, correspondant à deux conditions différentes.

L'une correspond à un système isolé dont l'énergie $E$ est fixée. C'est la distribution microcanonique. Elle postule que les $g(E)$ états d'énergie $E$ sont également probables (hypothèse ergodique). Par conséquent :

$p_{i}=1 / g\left(E_{i}\right)$, si $E_{i}=E ; p_{i}=0$, si $E_{i} \neq E$
L'autre distribution est valable pour un système en équilibre avec un très grand système (thermostat) avec lequel il a un très faible contact thermique. C'est la distribution canonique :

$$
p_{i}=\exp \left(-\beta E_{i}\right) / \sum_{j} \exp \left(-\beta E_{j}\right)
$$

où $\beta=1 / k_{B} T, T$ est la température du thermostat et $k_{B}$ la constante de Boltzmann.

À partir de (1) ou (2) on peut, en principe, calculer les fonctions thermodynamiques si on connait les interactions, c'est-à-dire le hamiltonien du système. Les physiciens de la matière condensée préferent utiliser la distribution canonique (2). Les astrophysiciens ne l'aiment guère, car leurs énormes objets seraient bien en peine de trouver un thermostat. Mais les deux distributions sont souvent équivalentes, comme nous allons le voir.

\section{Du canonique au microcanonique et vice versa}

De (2), on peut déduire la valeur moyenne canonique $\langle X\rangle_{c}(T)$ d'une quantité $X$, qui prend la valeur $X_{i}$ dans l'état $i$ :

$$
\langle X\rangle_{c}=\frac{\sum_{i} X_{i} \exp \left(-\beta E_{i}\right)}{\sum \exp \left(-\beta E_{i}\right)}
$$

C'est une fonction continue de $T$ pour tout système fini. En faisant $X_{i}=E_{i}$ dans (3a), on montre assez facilement que l'énergie interne canonique $U=\langle X\rangle_{\text {c }}$ est une fonction croissante de la température T. Par suite, la capacité calorifique $C=d U / d T$ est positive.

Pour un grand système, on peut récrire les sommes dans (3a) comme des intégrales sur l'énergie, dans lesquelles apparaît la valeur moyenne microcanonique $\langle X\rangle_{m c}$ :

$$
\langle X\rangle_{c}=\frac{\int\langle X\rangle_{m c} e^{S(E) / k_{B}-\beta E} d E}{\int e^{S(E) / k_{B}-\beta E} d E}=\frac{\int\langle X\rangle_{m c} e^{-\beta F(E)} d E}{\int e^{-\beta F(E)} d E}
$$

où $\exp \left[S(E) / k_{B}\right]$ est la densité d'états (à un facteur de normalisation près), ce qui définit l'entropie $S(E)$. En général, l'énergie libre $F(E)$ a un minimum et l'exponentielle $\exp [-\beta F(E)]$ a un maximum très aigu pour une valeur unique $E_{M} \cdot(3 \mathrm{~b})$ se réduit alors à $\langle X\rangle_{m c}\left(E_{M}\right)$ : les distributions canoniques et microcanoniques sont équivalentes. 


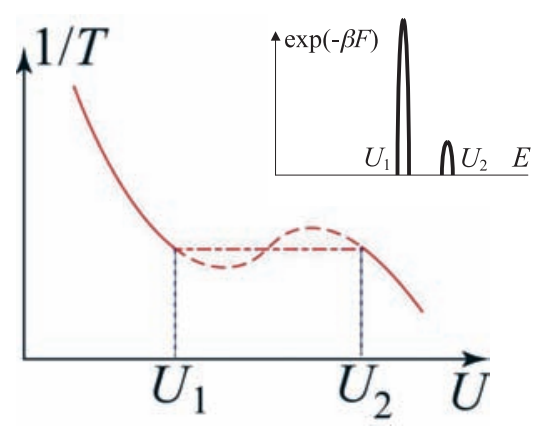

Figure 1. Transition de phase dans un grand système quand les deux phases ont des énergies différentes $\mathrm{U}_{1}$ et $\mathrm{U}_{2}$. Entre $\mathrm{U}_{1}$ et $\mathrm{U}_{2}$, pour des interactions à courte portée, la fonction $\mathrm{T}(\mathrm{U})$ tend vers un segment de droite (tirets et points). Pour des interactions à longue portée, la chaleur spécifique dU/dT peut devenir négative (tirets) dans le cas microcanonique. En insert, les deux maxima de la probabilité au voisinage d'une transition de phase du premier ordre. Â $T_{C}$, les deux maxima deviennent égaux.

Les quantités qui apparaissent naturellement sont l'énergie libre $F=E-T S$ et la température $T$ dans le premier cas, l'énergie interne $U$ et l'entropie $S$ dans le second, mais le changement de variables est sans problème, puisque $T=d U / d S$ et $F=U-T S$.

Il peut toutefois arriver que $\exp [-\beta F(E)]$ n'ait pas un, mais deux maxima aigus (insert de la figure 1). Cela arrive quand il y a une transition d'une phase 1 d'énergie $U_{1}$ à une phase 2 d'énergie $U_{2}$ à une certaine température $T_{c}$. Un exemple est donné par un fluide qui subit la liquéfaction ou la vaporisation à pression constante ; il faut alors modifier un peu les formules, faisant notamment apparaître dans l'exposant de (3b) l'enthalpie libre $E-T S+P V$ au lieu de l'énergie libre $E$ - TS. Rappelons que le refroidissement du fluide à volume constant mène à la coexistence de deux phases dans un intervalle fini de température, sans discontinuité de l'énergie ou d'autres quantités.

C'est donc une transition du premier ordre à volume constant, avec discontinuité de l'énergie $U_{1}$ à $U_{2}$, que nous allons désormais considérer. Dans la statistique canonique, cette discontinuité n'existe qu'à la limite $N \rightarrow \infty$, puisque (3a) est une fonction continue de $T$ pour $N$ fini. La limite de la fonction $T(U)$ est une fonction continue, non décroissante, avec un palier à $T_{c}$ entre $U_{1}$ et $U_{2}$ (fig. 1). Ceci est vrai pour la distribution canonique, avec des interactions à courte ou à longue portée.

Tout ce qui est dit ici est, sur le fond, en accord avec les manuels, même si ce n'y est pas toujours écrit explicitement et si la forme peut être différente.

Ainsi, la positivité de la capacité calorifique est-elle démontrée par Landau et Lifshitz [1] sans passer par la formule de Gibbs (2), mais en utilisant seulement l'équilibre avec le "milieu extérieur " que nous avons appelé «thermostat ». Si maintenant on veut traiter une transition du premier ordre avec discontinuité d'énergie dans le cas microcanonique, l'aide des manuels n'est plus très efficace. Nous rappellerons d'abord le cas des interactions à courte portée, auxquelles est consacré le reste de cette section. Il est facile de voir que dans la limite $N \rightarrow \infty$, la fonction $T(U)$ dans le cas microcanonique est alors la même que dans le cas canonique, avec une partie horizontale entre $U_{1}$ et $U_{2}$ (fig. 1). La chaleur spécifique est également la même, donc positive. Plus généralement, la plupart des manuels considèrent explicitement ou implicitement que les distributions canonique et microcanonique sont équivalentes pour des interactions à courte portée, au moins dans la limite $N \rightarrow \infty$.

Il y a pourtant une différence qui, dans le cas d'interactions à longue distance, aura des conséquences capitales. Dans le cas canonique, les énergies comprises entre $U_{1}$ et $U_{2}$ sont interdites pour un grand système. $\mathrm{Au}$ contraire, on peut imposer à l'énergie d'un système isolé (microcanonique) n'importe quelle valeur comprise entre $U_{1}$ et $U_{2}$, en le partageant en un domaine de la phase 1 et un domaine de la phase 2, séparés par une paroi. Celle-ci a une énergie $U_{W}$ proportionnelle, pour un échantillon tridimensionnel, à $N^{2 / 3}$. Si $N$ tend vers l'infini, cette énergie est négligeable par rapport à $U_{1}-U_{2}$, de sorte qu'elle ne joue pas un grand rôle dans l'analyse microcanonique. Mais, dans la probabilité canonique (2), elle introduit un facteur de la forme $\exp \left[-\right.$ const. $\left.N^{2 / 3} / T\right]$, de sorte que les états

\section{Interactions à longue et à courte portée}

Une interaction entre deux particules sera dite à longue portée si l'énergie décroît moins vite avec la distance $r$ qu'une exponentielle exp $(-\kappa r)$, par exemple comme une fonction puissance $1 / r^{\alpha}$. Remarquons toutefois que si $\alpha$ est trop grand, le système est extensif, comme on le montre à la section 5. Ainsi, les interactions de Van der Waals $(\alpha=6)$ ne détruisent pas l'extensivité.

Les interactions coulombiennes détruisent l'extensivité dans le noyau, qui possède une charge électrique, mais non en physique de la matière condensée, où la charge totale est nulle et où les charges locales sont compensées par une charge d'écran de signe opposé (voir la section 4).

comportant une paroi ont une probabilité pratiquement nulle pour un grand système. Comme l'écrit Gross [6], la statistique microcanonique est "plus riche ", puisqu'elle autorise des états qui sont interdits dans l'ensemble canonique. Mais il est excessif de conclure, comme le fait cet auteur, « qu'une transition de phase de premier ordre... est traitée correctement uniquement par la statistique microcanonique ». La statistique canonique n'interdit d'ailleurs pas la coexistence de deux phases comme, par exemple, l'eau liquide et sa vapeur, à volume fixé.

\section{Interactions à longue portée ayant un intérêt physique}

Il s'agit des interactions gravitationnelles, coulombiennes et hydrodynamiques, importantes en astronomie, en physique nucléaire et en mécanique respectivement, comme l'illustre la figure 2. Rappelons quelques propriétés de ces interactions.

L'interaction gravitationnelle (ou "gravité ") est un potentiel attractif proportionnel à $1 / r$ entre deux masses à distance $r$. Le coefficient de proportionnalité est faible, de sorte que dans un laboratoire, l'interaction gravitationnelle entre les atomes d'un gaz ou d'un liquide est négligeable. La pesanteur ne l'est pas, mais il est facile d'en tenir compte. En revanche, la gravitation introduit des complications essentielles à l'échelle d'une galaxie, ou même d'un astre: l'écroulement gravitationnel, qui finit par transformer une étoile de masse supérieure à un certain seuil en trou noir, est une manifestation spectaculaire de la non extensivité.

L'interaction coulombienne, également en $1 / r$, est bien différente, puisque répulsive entre charges de même signe. Aux échelles de longueur qui nous sont familières, toute charge électrique est généralement compensée par des charges électriques voisines de signe opposé, de sorte que l'interaction coulombienne donne naissance à des interactions chimiques attractives, et à courte portée. Le cas d'un noyau atomique est différent puisqu'il est chargé positivement. Son énergie est donnée approximativement par la formule de Bethe-Weizsächer [3], qui contient un terme non extensif dû à la répulsion coulombienne, proportionnel à $Z^{2} / A^{1 / 3}$, où $Z$ est le nombre de protons et $A$ le nombre de nucléons. Ce terme explique l'instabilité des noyaux trop lourds. 
La turbulence bidimensionnelle [7] constitue une application récente inattendue de la mécanique statistique non extensive. Les «particules » sont alors des tourbillons. La longue portée de l'interaction vient de la décroissance de la vitesse en $1 / r$ à distance $r$ d'un tourbillon. On trouve cette situation à la surface de la planète Jupiter [8].

\section{Modèles académiques d'interaction à longue portée}

Pour ne pas affronter trop de difficultés à la fois, il est bon de considérer des modèles non réalistes, où certaines de ces difficultés sont exclues artificiellement [9]. Ainsi, au lieu de particules mobiles, nous considérerons un réseau rigide aux $N$ points $\mathbf{R}_{i}$ duquel sont placées des variables $S_{i}=1$ ou -1 (spins). Particulièrement populaire est le modèle d'Ising qui associe une énergie d'interaction $-J\left(r_{i j}\right) S_{i} S_{j}$ à une paire de spins à distance $r_{i j}$. Supposons : $J(r)=C / r^{\alpha}$, où $C$ et $\alpha$ sont positifs. À basse température, tous les spins ont le même signe et l'énergie par spin est proportionnelle, en champ nul, à :

$$
\int \frac{r^{2} d r}{r^{\alpha}} \approx \text { Const }+L^{3-\alpha} /(3-\alpha)
$$

Le modèle est non extensif si l'énergie par spin dépend de la taille de l'échantillon, donc si le second terme l'emporte sur le premier, soit : $\alpha \leq 3$.

L'énergie $U / N$ par spin est alors proportionnelle à $L^{3-\alpha}$ d'après (4). Il y a lieu d'introduire aussi un champ magnétique, mais celui-ci devra être capable d'entrer en compétition avec (4), ce qui impose, si $\alpha<3$, qu'il soit proportionnel à $L^{3-\alpha}$, soit $N^{1-\alpha / 3}$.

Le modèle d'Ising à interactions indépendantes de la distance $(\alpha=0)$

Ce problème très artificiel n'a pas d'autre interêt que d'être exactement soluble. L'énergie ne dépend en effet que de l'aimantation réduite :

$m=(1 / N) \sum S_{i}$ comprise entre -1 et 1.
Elle peut s'écrire, dans un champ magnétique $N h$ :

$$
U=-(J / 2) N^{2}(m+h / J)^{2}-N^{2} h^{2} /(2 J)
$$

L'entropie est pour $N$ grand :

$S / k_{B}=N \ln 2-(N / 2)$

$$
[(1-m) \ln (1-m)+(1+m) \ln (1+m)]
$$

Elle est proportionnelle à $N$, alors que l'énergie (5) est proportionnelle à $N^{2}$.

La valeur $m(T)$ de $m$ à l'équilibre canonique est donnée par le minimum de $F(m)=U(m)-T S(m)$. C'est un exercice classique, car il correspond à l'approximation du champ moyen du modèle d'Ising habituel (à interactions entre premiers voisins), à ceci près que le facteur $N$ habituel est devenu $N^{2}$ dans (5). Si on fait varier $T$ à champ $h$ constant, il n'y a de transition que quand $h=0$, et elle est continue (ou "du second ordre »). Si on augmente $h$ à $T$ constant, il y a une transition du premier ordre pour $h=0$, où l'aimantation passe de $-m$ à $m$, mais l'énergie (5) n'a pas de discontinuité. En l'absence d'une telle discontinuité, nous savons par le raisonnement de la section 3 que les distributions canonique et microcanonique sont équivalentes.

Cette absence de bande d'énergie interdite est liée à la symétrie du problème : l'énergie libre U-TS ne peut avoir deux minimas égaux 1 et 2 que si $h=0$; mais pour $h=0, U$ et $S$ sont des fonctions paires de $m$, de sorte que $m_{1}=-m_{2}$ et $U_{1}=$ $U_{2}$. Pour examiner les conséquences d'une discontinuité de l'énergie, comme nous l'avons fait pour des interactions à courte portée dans la section 3 , nous allons considéré un cas moins symétrique.

\section{Capacité calorifique négative}

Un modèle intéressant s'obtient en remplaçant les interactions à deux corps du modèle d'Ising par des interactions à trois spins (ici encore, indépendantes de la distance). La formule (5) est alors remplacée par :

$$
U=-K N^{3} m^{3}-K h N^{3} m
$$

où le champ magnétique $N^{2} h$ doit être proportionnel à $N^{2}$ pour pouvoir équilibrer les interactions entre spins. L'entropie est encore donnée par (6). L'équilibre canonique d'un grand système doit minimiser l'énergie libre $F(m)=U(m)-T S(m)$. Pour $h>0$, ce minimum est obtenu pour une valeur $m(T)$ qui croît de 0 à 1 quand $T / N$ décroît de l'infini à 0 . Si $h$ n'est pas trop grand, $m(T)$ a une discontinuité pour une certaine température de transition $T_{c}$. À $T_{c}, F(m)$ a deux minima égaux, 1 et 2 (fig. 3). Ils correspondent à des valeurs différentes, $U_{1}$ et $U_{2}$, de (7). Il n'y a pas de valeur de $T$ telle que $U(m(T))$ soit située entre ces deux valeurs. Nous sommes donc dans le cas de la figure 1.

Pourtant, si le système est isolé, il est possible de lui donner une aimantation arbitraire comprise entre $m_{1}$ et $m_{2}$. Quand $m$ varie entre $m_{1}$ et $m_{2}$, l'énergie $U(m)$ varie entre $U_{1}$ et $U_{2}$ d'après (7), l'entropie $S(m)$ entre $S_{1}$ et $S_{2}$ d'après (6), et l'on peut définir une température par la formule habituelle $T=d U / d S$, où la dérivée est à volume et nombre de particules constants. On obtient ainsi la ligne en tirets de la figure 1. La température n'est plus une fonction croissante de $U$ ! Dans la zone où elle est décroissante, la capacité calorifique $C=d U / d T$ est négative !

Cette propriété d'avoir une capacité calorifique microcanonique négative est un paradoxe amusant, dont les conséquences physiques sont limitées : ce qu'on mesure dans les laboratoires est la capacité calorifique canonique, qui est positive.

L'interaction gravitationnelle fournit un exemple non académique (mais bien plus difficile à traiter) de chaleur spécifique négative ${ }^{(1)}$.
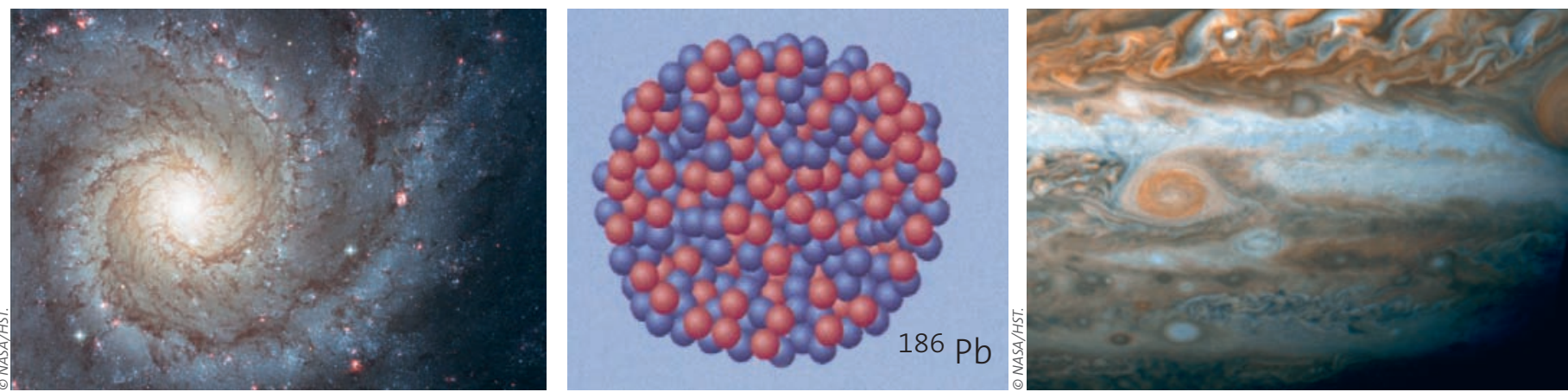

Figure 2 : Quelques systèmes régis par des interactions à longue portée. (a) Galaxie (interaction gravitationnelle). (b) Noyau atomique (interaction coulombienne entre protons représentés par les sphères rouges). (c) Surface de la planète Jupiter (turbulence bidimensionnelle). 


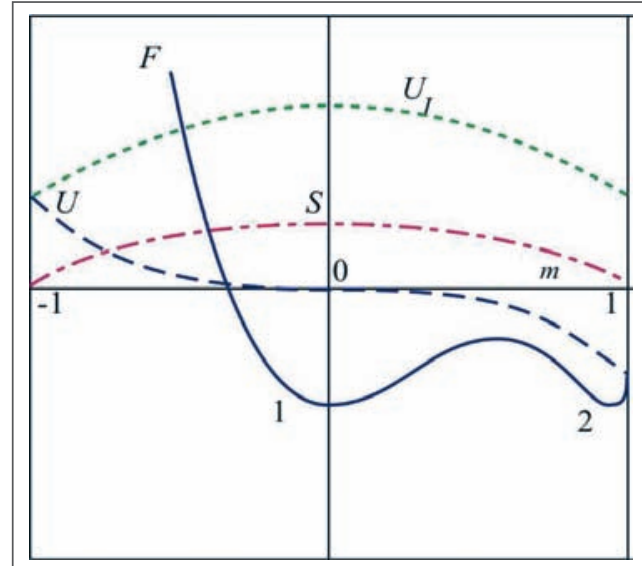

Figure 3. Énergie U (tirets) et entropie S (traits points) en fonction de l'aimantation $\mathrm{m}$, dans le cas du modèle non extensif défini par (6) et (7) avec $h$ voisin de 0 mais légèrement positif. L'énergie libre $\mathrm{F}=\mathrm{U}$-TS (ligne continue) a deux minima dégénérés 1 et 2 pour une valeur critique de la température correspondant à deux valeurs $\mathrm{m}_{1}$ et $\mathrm{m}_{2}$ de $\mathrm{m}$. Les unités de l'axe vertical sont différentes pour les trois courbes, et dépendent du nombre $\mathrm{N}$ de spins. La courbe supérieure (pointillée) montre la forme symétrique de l'énergie $U_{1}$ du modèle d'lsing pour la valeur critique $\mathrm{h}=0$ du champ.

\section{Interactions dépendant de la distance}

Au paragraphe précédent, nous avons considéré des interactions indépendantes de la distance $r$. Le cas d'interactions en $1 / r^{\alpha}$ avec $0<\alpha<3$ est bien plus compliqué. Si on considère, par exemple, le modèle d'Ising, l'une des difficultés est que l'influence de la surface se fait sentir dans tout l'échantillon, l'intégrale $\mathrm{d}^{3} \boldsymbol{r}^{\prime} /\left|\boldsymbol{r}-\boldsymbol{r}{ }^{\prime}\right|^{\alpha}$ variant continûment du centre au bord. L'état du système est donc décrit par un champ $m(r)$, et pas du tout par un seul paramètre d'ordre $m$ comme dans les équations (5), (6) et (7). C'est la nature inhomogène des états qui nous a amenés à parler de capacité calorifique, et non de chaleur spécifique, expression appropriée pour un état homogène.

Un calcul précis comme dans la section précédente serait difficile, mais on s'attend à trouver, dans certains cas, une bande d'énergie interdite dans le cas canonique, entre deux valeurs $U_{1}$ et $U_{2}$. Dans le cas microcanonique, cette bande n'est pas interdite, et un postulat plausible est que la température $T(U)$ est une fonction continue et dérivable pour $U_{1}<U<U_{2}$. Comme $T\left(U_{1}\right)=T\left(U_{2}\right)$, il faut, soit que $d T / d U$ soit négative pour certaines valeurs, soit que $d T / d U=0$ partout entre $U_{1}$ et $U_{2}$, comme c'est le cas pour des interactions à courte portée, ainsi qu'on l'a vu dans la section 3. Pour des interactions à longue portée, il n'y a aucune raison qu'il en soit ainsi : $T(U)$ doit suivre la courbe en tirets de la figure 1, et la capacité calorifique $C=d U / d T$ doit être négative pour certaines valeurs de $U$.

\section{Conclusion}

La lecture de plusieurs articles récents peut susciter une certaine confusion. La façon correcte de voir les choses semble être la suivante :

\section{I - Particularités des interactions à longue portée non extensives}

a) Elles sont implicitement exclues de bien des manuels de thermodynamique.

b) L'état d'équilibre n'est généralement pas homogène.

c) Il n'y a pas de " limite thermodynamique ", c'est-à-dire qu'on ne peut faire tendre $N$ vers l'infini en maintenant les quantités intensives (température, densité, aimantation...) fixées [10].

\section{II - Statistique canonique}

a) La distribution canonique décrit correctement un système fini $\mathrm{S}$ (petit ou grand) en équilibre avec un thermostat, quelles que soient les interactions (à longue ou courte portée) à l'intérieur du système $S$ [11]. Des précautions sont cependant nécessaires : "Dans des systèmes comportant des interactions à longue portée, l'ensemble canonique ne décrit pas les fluctuations d'un petit sous-système. Toutefois il peut décrire les fluctuations du système entier couplé à un thermostat avec un couplage infiniment faible." [12] Cette analyse nuancée contraste avec l'affirmation radicale et probablement excessive de Combes et Robert [13] que "[for non-extensive systems]... the canonical approach has no justification at all", ou avec le réquisitoire de Gross [6] contre des "errors of conventional canonical thermostatistics", que le lecteur a du mal à identifier.

b) La capacité calorifique canonique est positive dans tous les cas.

c) La distribution canonique est très commode, mais elle n'est évidemment pas applicable en astrophysique, alors que c'est là que les interactions à longue portée sont les plus importantes.

\section{III - Statistique microcanonique}

a) Elle permet des états de la matière qui sont interdits par la distribution canonique [6, 14].

b) Dans le cas d'interactions à courte portée, la chaleur spécifique d'un système est la même que pour la distribution canonique. Elle est donc positive. Toutefois, certains états avec coexistence de phases sont permis, alors qu'ils sont impossibles avec la distribution canonique. c) Dans le cas d'interactions à longue portée, la capacité calorifique peut être négative. Notons que même la distribution microcanonique est également sujette à des hypothèses restrictives, telle que l'ergodicité. Un manque éventuel d'ergodicité peut avoir de lourdes conséquences $[15,16]$. On peut donc avoir à considérer des distributions qui ne sont ni canoniques ni microcanoniques.

\section{Références}

[1] L. Landau, E. Lifshitz, Physique statistique (Mir, Moscou, 1967).

[2] T. Dauxois et al. (Eds.),

"Dynamics and Thermodynamics of Systems with Long Range Interactions", Lecture Notes in Phys., vol. 602 (Springer-Verlag, Berlin, 2002).

[3] K. F. von Weizsäcker, Zeitschrift für Physik 96 (1935) 431.

Voir aussi le site Internet: http://fr.wikipedia.org/wiki/ Formule_de_Weizsäcker

[4] W. Thirring, Z. Phys. 235 (1970) 339.

[5] F. Reif, "Physique statistique ", Berkeley, Cours de physique, vol. 5 , traduit par P. Turon (Armand Colin, 1994).

[6] D.H.E. Gross, C. R. Physique 7 (2006) 311.

[7] F. Bouchet, J. Sommeria,

J. Fluid. Mech. 464 (2002) 165.

[8] A. Boischot et al., "Jupiter" dans l'Encyclopaedia Universalis (2005).

[9] J. Barré et al., J. Stat. Phys. 119 (2005) 677.

[10] J. Barré, F. Bouchet, C.R. Physique 7 (2006) 414.

[11] W. Thirring, $A$ course in Mathematical physics (Springer, Heidelberg, 1991).

[12] F. Bouchet, J. Barré, J. Stat. Phys. 118 (2005) 1073.

[13] F. Combes, R. Robert,

C. R. Physique 7 (2006) 307.

[14] P.H. Chavanis, Int. J. M. Phys. B 20 (2006) 3113.

[15] M.L. Chabanol, F. Corson, Y. Pomeau, Europhys. Lett. 50 (2000) 148.

[16] W. Thirring, H. Narnhofer, H. A. Posch, Phys. Rev. Lett. 91 (2003) 130601.

(1) Un exemple classique de capacité calorifique négative est celui d'une étoile qui a épuisé son combustible nucléaire en émettant de l'énergie de radiation, elle se contracte et sa température augmente [14]. Chavanis donne une démonstration générale de la capacité calorifique négative des systèmes "autogravitants " à partir du théorème du viriel, dont on trouve une démonstration appropriée à ces systèmes sur le site :

http://scienceworld.wolfram.com/ physics/VirialTheorem.html. 